\title{
A ETNOMATEMÁTICA NO PROCESSO EDUCATIVO: O ENSINO DE ESPAÇO E FORMA PARA OS ALUNOS DOS ANOS INICIAIS DO ENSINO FUNDAMENTAL
}

ETHNOMATHEMATICS IN THE EDUCATIONAL PROCESS: TEACHING SPACE AND FORM FOR STUDENTS IN THE FIRST YEARS OF ELEMENTARY SCHOOL

\author{
Débora Renata Marques Muniz ${ }^{1}$ \\ Luciano Tadeu Corrêa Medeiros ${ }^{2}$ \\ Paulo Henrique Fernandes de Souza Dias ${ }^{3}$ \\ Waléria Néri Oliveira Ribeiro ${ }^{4}$
}

RESUMO: Este ensaio apresenta algumas das vivências e experiências como discente do Curso de Pedagogia, da Faculdade de Educação, da Universidade Federal do Pará - UFPA. O objetivo é discutir sobre os assuntos ministrados nas disciplinas referentes ao ensino da matemática nos Anos Iniciais do Ensino Fundamental. Para tanto, elegemos o Componente Curricular obrigatório Abordagens Teórico-metodológicas da Matemática Escolar e destacamos a tendência Etnomatemática e sua aplicabilidade para o ensino de Espaço e Forma para os alunos dessa etapa de ensino da Educação Básica. Utilizou-se a abordagem qualitativa, que contou com uma pesquisa bibliográfica de texto dos autores D’Ambrósio (2005); Fiorentini (1994); Smole (2012); Rodrigues (2005); e Rodrigues (2019). Desenvolveu-se, ainda, uma análise das atividades realizadas durante a disciplina. Os resultados indicam a importância de se conhecer a tendência Etnomatemática e seus métodos, visto que a mesma apresenta uma importante possibilidade do ensino dos saberes da Matemática para os alunos dos Anos Iniciais do Ensino Fundamental.

Palavras-chave: Anos Iniciais. Espaço e Forma. Etnomatemática. Processo educativo.

ABSTRACT: This essay presents some of the experiences as a student of the Pedagogy Course, at the Faculty of Education, at the Federal University of Pará - UFPA. The objective is to discuss the subjects taught in the subjects related to the teaching of mathematics in the Early Years of Elementary School. To this end, we chose the mandatory Curricular Component Theoreticalmethodological approaches to School Mathematics and highlighted the Ethnomathematics trend and its applicability to the teaching of Space and Form for students in this stage of Basic Education. The qualitative approach was used, which included a bibliographic text search by the authors D'Ambrósio (2005); Fiorentini (1994); Smole (2012); Rodrigues (2005); and Rodrigues (2019). An analysis of the activities carried out during the discipline was also developed. The results indicate the importance of knowing the Ethnomathematics trend and its methods since it

\footnotetext{
${ }^{1}$ Universidade Federal do Pará - UFPA, e-mail: debora.muniz@ifch.ufpa.br.

${ }^{2}$ Universidade Federal do Pará - UFPA, e-mail: luciano.medeiros@iced.ufpa.br.

3 Universidade Federal do Pará - UFPA, e-mail. paulo.dias@lasalle.org.br.

4 Universidade Federal do Pará - UFPA, e-mail.waléria_neri@hotmail.com.
} 
presents an important possibility of teaching the knowledge of Mathematics to students of the Early Years of Elementary School.

Keywords: Initial Years. Space and Form. Ethnomathematics. Educational process.

\section{INTRODUÇÃO}

Este trabalho é resultado de estudos realizados na disciplina Abordagens Teóricometodológicas da Matemática Escolar, ofertada pelo Curso de Pedagogia, da Faculdade de Educação, da Universidade Federal do Pará - UFPA. Nosso objetivo é refletir sobre o processo de ensinoaprendizagem de Matemática nos Anos Iniciais do Ensino Fundamental pautado na Etnomatemática, no qual enfatizamos estudos relativos a Espaço e Forma.

Espaço e Forma é um dos blocos de conteúdos matemáticos propostos pelos Parâmetros Curriculares Nacionais - PCN, documentos que orientavam a construção do currículo escolar até o ano de 2017, quando foi implantada a Base Nacional Comum Curricular - BNCC. O documento apresentava, ainda, estudos de Geometria que envolvem um conjunto de conceitos e procedimentos essenciais para a resolução de problemas e na representação e exploração de objetos presentes no cotidiano, o que permite ao aluno fazer correlações entre a Matemática, o mundo físico e outros campos de conhecimentos.

$\mathrm{Na}$ BNCC, Espaço e Forma, está presente dentro da Geometria como conhecimento essencial a ser ensinado. A proposta desse documento é propiciar aos alunos a apreensão de saberes e aprendizados que possibilitem a resolução de problemas que permeiam o cotidiano dos educandos, relacionando-os à Matemática Escolar - formas geométricas, localização de pessoas e objetos, dentre outros. Ressaltamos, que a BNCC, ao contrário dos PCN, apresenta-se no contexto atual como documento normativo, vigente desde o ano de 2017 por meio da Resolução CNE/CP n. 2.

Para a realização deste trabalho, propusemo-nos às seguintes questões norteadoras: Como a Tendência Etnomatemática colabora para o processo de ensino e aprendizagem da matemática nos anos iniciais do Ensino Fundamental? Para tanto, utilizamos como critério investigativo a abordagem qualitativa, através de uma pesquisa bibliografia sobre o assunto e as vivências durante a disciplina ATM de Matemática Escolar. Desse componente curricular, elegemos três atividades realizadas em sala de aula, orientadas pelo docente que ministrou a referida disciplina, cujos resultados encontram-se em formato de imagens, como maneira de dialogar sobre as provocações suscitadas em sala na relação aluno e professor acerca da Etnomatemática no espaço escolar, compreendendo seus significados e sua relevância na Geometria, em especial no estudo sobre Espaço e Forma. O referencial teórico foi baseado nos textos de D’ Ambrósio (2005); Fiorentini 
(1994); Smole (2012); Rodrigues (2005); e Rodrigues (2019). Também foram utilizados documentos legais, como a BNCC e os PCN de matemática.

O texto está estruturado em três seções. Na primeira seção, abordamos questões relativas ao bloco de conteúdo matemático Espaço e Forma de acordo com os PCN de Matemática, que, na atual BNCC, encontra-se como conteúdo de Geometria. Na segunda seção, trazemos olhares acerca da Etnomatemática como inspiradora em processos de ensino e aprendizagem no estudo de Espaço e Forma nos anos iniciais do Ensino Fundamental, procurando estabelecer diálogos com o que foi estudado e discutido na disciplina ATM de Matemática Escolar sobre esses assuntos. Por fim, na última seção, apresentamos as considerações finais como culminância do referido trabalho, a importância de se conhecer essa tendência por parte de pedagogos (as) em formação, o que será de grande auxílio em suas práticas docentes nos Anos Iniciais quando estiverem atuando na sala de aula dessa etapa de ensino.

\section{A GEOMETRIA NOS ESTUDOS DE ESPAÇO E FORMA}

Ao compreender o mundo físico através das ações humanas desenvolvidas no cotidiano e na vida prática, os sujeitos tornam-se seres sociais aptos para a resolução de problemas comuns de seu dia a dia. Ao nos deparamos com situações que envolvem questões geométricas presentes em objetos, lugares e outros elementos de nossa realidade física, percebemos, a partir de nossa visão tridimensional, como esses elementos se assemelham às figuras geométrica apresentadas pela matemática e como se encontram nas formas de elementos da natureza. As figuras geométricas também se encontram presentes em outras áreas de conhecimento, onde o uso dessas figuras se torna bem mais comum, a exemplo da Arte, Arquitetura, Geografia, assim por diante (BRASIL, 1997; BRASIL; 2017; FELIX; AZEVEDO; 2015).

De acordo com Felix e Azevedo (2015), a geometria possibilita uma leitura de mundo que não deve estar relacionado somente ao uso social, mas, precisa ser trabalhada sua terminologia própria, conforme a cognição do aluno e a etapa de ensino em que se encontra. Conforme nos alerta Félix e Azevedo (2015, p. 3), ressaltamos que o "objetivo de ensinar a geometria aos alunos de $\mathrm{I}^{\mathrm{O}}$ a $5^{\mathrm{O}}$ ano está ligado ao sentido de localização, reconhecimento de figuras, manipulação de formas geométricas, representação espacial e estabelecimento de propriedades".

Nesse sentido, na geometria, os alunos aprendem, por meio da observação, manipulação e representação dos espaços e objetos geométricos, a construir ideias, formular hipóteses, e, a partir da visualização ou manipulação, produzir representações escritas e mentais acerca de situações-problemas, pode-se dizer, portanto, que: 
Os conceitos geométricos constituem parte importante do currículo de Matemática no ensino fundamental, porque, por meio deles, o aluno desenvolve um tipo especial de pensamento que lhe permite compreender, descrever e representar de forma organizada, o mundo em que vive. (BRASIL, 1997, p.39).

Nos PCN de matemática (BRASIL, 1997), o estudo de Espaço e Forma está elencado em uma série de conteúdos que o educador precisa ensinar ao aluno, entre eles, destacam-se: localização de pessoas ou objetos no espaço, observação de formas geométricas presentes na natureza, comparar e estabelecer relações entre o objeto geométrico - esféricos, cilíndricos, cônicos, etc. - e o espaço físico, construir e representar formas geométricas, assim por diante. Já na BNCC (2017), uma das habilidades da unidade temática que trata de Geometria é saber relacionar as figuras geométricas planas e espaciais a objetos familiares do mundo físico, saber utilizar pontos de referências para a localização de pessoas e objetos, e associar a terminologia especifica desse conteúdo - círculo, quadrado, cilindros, esferas, direita, esquerda, em frente, atrás, em cima, em baixo. No documento, questões relacionas ao aprendizado de Espaço Forma encontram-se dentro da unidade temática denominada de Geometria, que considera este saber matemático como:

[...] um amplo conjunto de conceitos e procedimentos necessários para resolver problemas do mundo físico e de diferentes áreas do conhecimento. Assim, nessa unidade temática, estudar posição e deslocamentos no espaço, formas e relações entre elementos de figuras planas e espaciais pode desenvolver o pensamento geométrico dos alunos. Esse pensamento é necessário para investigar propriedades, fazer conjecturas e produzir argumentos geométricos convincentes. É importante, também, considerar o aspecto funcional que deve estar presente no estudo da Geometria: as transformações geométricas, sobretudo as simetrias. As ideias matemáticas fundamentais associadas a essa temática são, principalmente, construção, representação e interdependência. (BRASIL, 2017, p. 37I).

Nessa ótica, tanto Espaço e Forma nos PCN ou Geometria na BNCC são conteúdos a serem ensinados nos Anos Iniciais do Ensino Fundamental e, com isso, a prática docente deve atentar para que a construção do conhecimento matemático em sala de aula também seja útil para os educandos fora desse espaço, porém, isso depende da maneira como o professor irá trabalhar esse assuntos com as crianças, pois compreendemos que esses saberes precisam estar articulados às necessidade apresentadas no reconhecimento do mundo físico, trazendo como perspectiva possibilitar esses alunos a relacionar, experimentar, descrever e representar os conceitos geométricos dentro da sua própria realidade social (BRASIL, 1997; BRASIL, 2017; FELIZ; AZEVEDO, 2015). 


\section{ETNOMATEMÁTICA NO ENSINO DE GEOMETRIA: ESPAÇO E FORMA}

Nos estudos realizados na disciplina de ATM da Matemática Escolar, no curso de Pedagogia da Universidade Federal do Pará, foram abordados diferentes modos de ver e conceber o ensino de matemática no Brasil. Fiorentini (1995), em seus estudos, identificou seis tendências que ele classificou como: Tendência Formalista Clássica; Tendência Empírico Ativista; Tendência Formalista Moderna; Tendência Tecnicista e suas variações; Tendência Construtivista; e Tendência socioetnocultural. Em cada uma dessas tendências, existem formas diferenciadas no entendimento da matemática e suas finalidades de ensino, a forma como tais tendências percebem as relações entre professor e aluno e a relação ensino e aprendizagem, dentre outros pontos nessas relações (FIORENTINI, 1995).

A questão da Etnomatemática foi formulada e evidenciada na Tendência Socioetnocultural, em que a matemática é entendida como um conhecimento relacional, onde pesam as relações culturais que são estabelecidas por determinados grupos sociais, a exemplo dos quilombolas, pescadores, dentistas, agricultores, pedreiros, artesãos e outros mais. Essa tendência aponta que cada grupo apresenta um tipo de conhecimento matemático peculiar (FIORENTINI,1995; HAGE, 2016).

D’Ambrósio (1990, p.17) afirma que etnomatemática “é um programa que visa explicar os processos de geração, organização e transmissão de conhecimentos em diversos sistemas culturais e as forças interativas que os agem entre os três processos". A Etnomatemática pode ser vista como um campo de conhecimento intrinsecamente ligado a grupos culturais e a seus interesses comuns, procurando entender suas formas particulares de organização, onde o aprendizado dos sujeitos partícipes desses grupos tenha, nesse processo, uma percepção que considere suas culturas, hábitos e costumes.

A utilidade dessa tendência para a Matemática Escolar nos anos iniciais do Ensino Fundamental tem como uma das suas principais funções a contextualização dos saberes vividos para o ensino e aprendizado dos conteúdos matemáticos, dentre eles a Geometria, e com ela o aprendizado de Espaço e Forma.

Assim, por meio das discussões apresentadas na disciplina ATM de Matemática, do curso Pedagogia da UFPA, foi possível compreender a importância da apropriação teórica sobre as múltiplas tendências para o ensino da matemática para os alunos dos anos iniciais, das quais destacamos a Etnomatemática como uma das importantes tendências para as práticas educativas desses saberes nessa etapa de ensino. 


\section{TAREFAS REALIZADAS EM SALA DE AULA: ENTENDENDO A ETNOMATEMÁTICA}

Em relação à prática, o docente que ministrou a disciplina utilizou como estratégias de apropriação em sala de aula várias atividades sobre o estudo de Espaço e Forma, das quais utilizaremos quatro. Sendo elas: I) mapa da Cidade Universitária do Guamá; 2) descrição do trajeto de entrada e saída do local; 3) construção de um objeto geométrico tridimensional; 4) relação desse objeto com três objetos do mundo físico por meio de desenho.

$\mathrm{Na}$ sala de aula, foi proposto representar, por meio de mapa, a visão superior de uma parte do Campus Universitário, como apresentado na imagem abaixo:

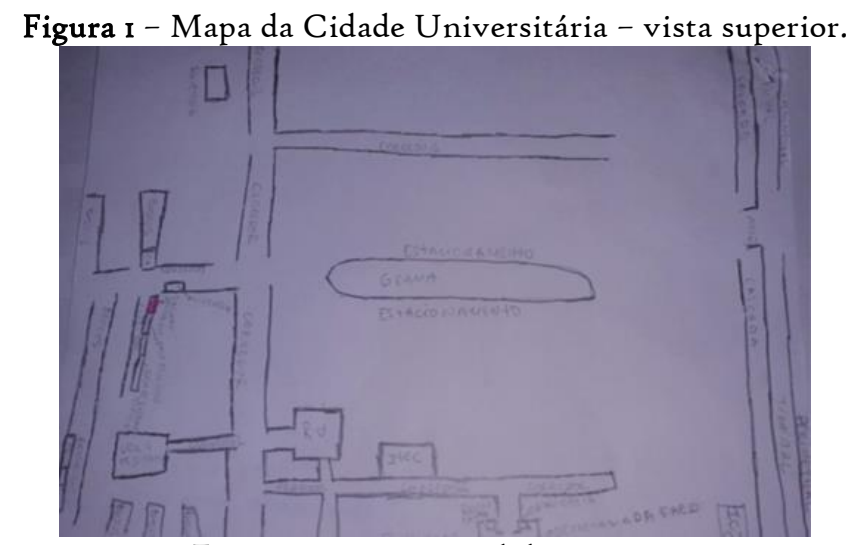

Por meio da confecção desse mapa, foi possível perceber a ideia sobre a localização de objeto e de pessoas a partir de uma visão superior desse espaço, o que deve ser entendido como algo presente na subjetividade dos sujeitos, portanto, são ensinamentos imprescindíveis para que os alunos dos anos iniciais possam desenvolver compreensão do seu espaço físico, através de sua percepção subjetiva desse espaço.

Outra tarefa solicitada pelo discente consistiu em utilizarmos as localizações a partir da representação do mapa e propormos orientações para que uma pessoa conseguisse chegar aos seguintes espaços: a) Sala dos professores do Instituto de Ciências da Educação - ICED; Protocolo do ICED; c) Secretaria da Faculdade de Educação - FAED.

O docente orientou a composição desse roteiro de localização, que deveria possibilitar a pessoa a iniciar e finalizar seu trajeto tendo como ponto de referência o portão do campus 3 da Universidade. Na sequência, apresentamos a transcrição do roteiro de localização apresentado pelo docente e produzido pelos autores durante o desenvolvimento da atividade da disciplina ATM de Matemática. 
Iㅇ passo: Como ponto de partida a pessoa teria o portão do campus 3 ; $2^{-}$passo: a pessoa deveria seguir o corredor de entrada e dobrar a esquerda, seguir novamente no corredor e dobrar a direita. Essas coordenadas deveriam levar a pessoa a uma Livraria, que se encontra nesse local, dentro do espaço percorrido, em seguida, a pessoa deveria seguir no corredor da livraria e virar à esquerda e encontrar a localização da sala dos professores. $3^{\circ}$ passo: tendo como ponto de partida agora a sala dos professores, a pessoa deveria ser orientada a chegar no Restaurante Universitário - RU, para isso, deveria retornar ao corredor da sala dos professores, fazendo o caminho inverso, passando novamente em frente à Livraria que fica na esquina do corredor e dobrar à direita, seguir e dobrar novamente à direita, chegando ao RU. Agora tendo como ponto de partida o RU, a pessoa teria que passar em frente ao Instituto de Tecnologia - ITEC e chegar ao Instituto de Educação - ICED, para isso, ela deveria dobrar à esquerda e seguir no corredor onde se encontra o ITEC, continuar seguindo pelo mesmo corredor e dobrar à direita, chegando à localização do ICED. $4^{\circ}$ Passo: ao entrar no ICED, localizar do lado esquerdo a Faculdade de Educação - FAED e no lado direito a sala do Protocolo do ICED. $5^{\circ}$ Passo: Saindo do ICED, a pessoa teria que seguir retornando pelo corredor que entrou e dobrar à direita até chegar ao calçamento lateral da pista de entrada do campus, dobrar à esquerda e caminhar pela calçada até chegar ao estacionamento localizado em frente ao portão de entrada do campus 3, o que levaria a pessoa ao seu transporte, caso estivesse em seu veículo particular, ou saindo pelo portão, encontrar a sua frente o Terminal de ônibus Urbano localizado na entra do campus, caso precisasse se transportar através de um ônibus.

Observamos que, seja na confecção do mapa, ou na descrição do roteiro de localização, percebe-se que os discentes conseguem descrever e representar com detalhes elementos que compõem sua realidade social, que está ligada a sua rotina universitária, visto que as informações orientam a leitura do mapa, tanto na representação da posição dos locais apresentadas por meio de desenho, ou pela descrição do lugar em sua forma de apresentar pontos de referências, como biblioteca, restaurante universitário e outros.

Importa ressaltar que questões como a subjetividade do lugar representado e descrito são imaginaç̃̃es sobre o espaço, materializadas nas descrições ou no mapa. São subjacentes, mas expressam com clareza a localidade e mostram com precisão a compreensão do espaço, uma vez que esses fazem parte da realidade intrínseca à memória dos alunos, ligadas à sua afetividade e cotidiano dentro das relações de suas vidas de universitários.

Assim, essa atividade pode ser entendida pela ótica multicultural, interdisciplinar e transdisciplinar, tomando como base a Etnomatemática, pois possibilita aplicar essa tendência de 
ensino dentro do contexto social dos estudantes - multiculturalidade, permitindo ainda o diálogo com outros campos da ciência, como a Geografia - interdisciplinaridade, visto se tratar, da representação de um espaço geográfico. Também possibilita uma leitura antropológica e linguística do lugar - transdisciplinaridade, pois considera-se as subjetividades e o código linguístico desses sujeitos, sem deixar de lado os conteúdos pertinentes à Matemática Escolar (D’AMBRÓSIO, 2005).

Reconhecemos, portanto, que usar a Etnomatemática em sala de aula requer valorizar a cultura das várias comunidades existentes, no Brasil, por exemplo, das comunidades dos assentamentos rurais, ribeirinhas, quilombolas, das periferias da cidade, e de tantras outras que possuem, dentro de seu espaço, uma cultura peculiar, que deve ser considerada em toda sua dimensão simbólica, histórica e sociocultural (HAGE, 2016; D’ AMBRÒSIO, 2005).

Outra estratégia sobre as práticas educativas apresentada pelo docente sobre o conteúdo Espaço e Forma, em sala de aula, foi feita a partir da proposta de construção de um objeto geométrico tridimensional - cubo, cilindro, cone, etc. -, conforme podemos perceber na imagem, a seguir.

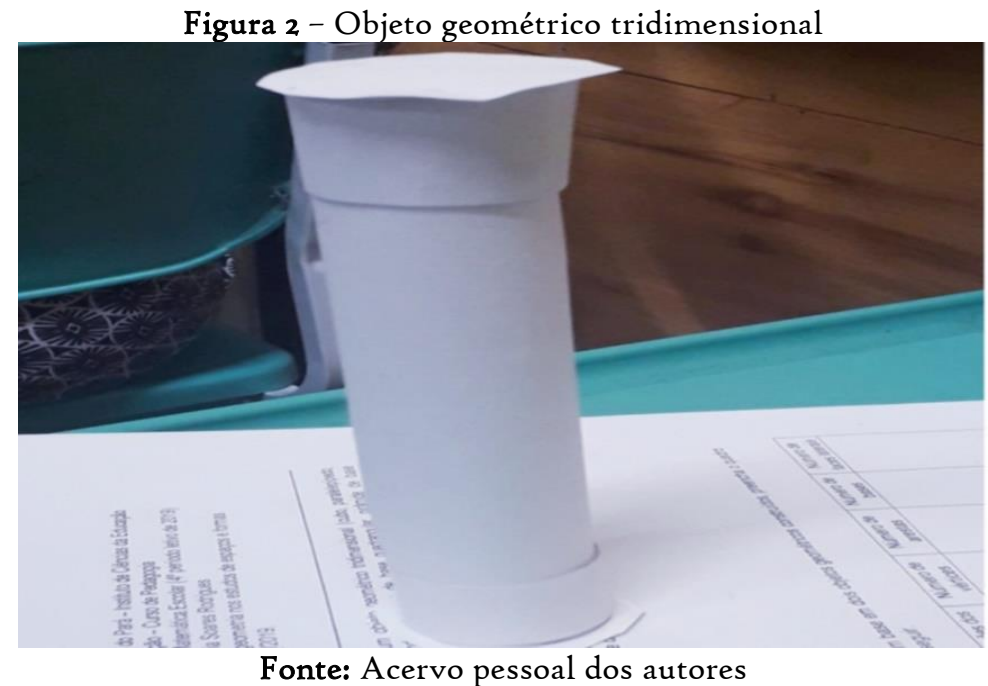

O cilindro, figura representada e confeccionada durante às aulas, mostra de que forma esse espacial geométrico pode se apresentar no cotidiano, o que nos permite compreender que o conhecimento matemático precisa ser ensinado às crianças dos Anos Iniciais mostrando de forma material os objetos que se apresentam com essa forma. Essa dinâmica é essencial para a compreensão dos alunos, como demonstrado pelo docente da disciplina ATM de Matemática. Nesse sentido, a utilização de materiais manipuláveis, como aponta Rodrigues (2019, p. 5), 
possibilita aos alunos (as) perceberem e estabelecerem: “[...] relações com diferentes formas existentes no seu dia a dia e os espaços que essas formas ocupam, possibilitando o conhecimento de conceitos geométricos".

Evidencia-se, que a utilização de matérias manipuláveis também está relacionada à Tendência Empírico-Ativista, visto que, sob a ótica dessa tendência, o aluno deve aprender a partir da prática, porém, ela não deixa de se relacionar com a Etnomatemática, pois, a construção e representação desse material manipulável relaciona-se com a vida prática dos sujeitos (FIORENTINI, I995; RODRIGUES, 2019).

A afirmação de Rodrigues (2019) coaduna com outra atividade proposta, que é representar graficamente três figuras que apresentem essa forma espacial geométrica no cotidiano.

Figura 3 - Objeto geométrico encontrado no mundo físico

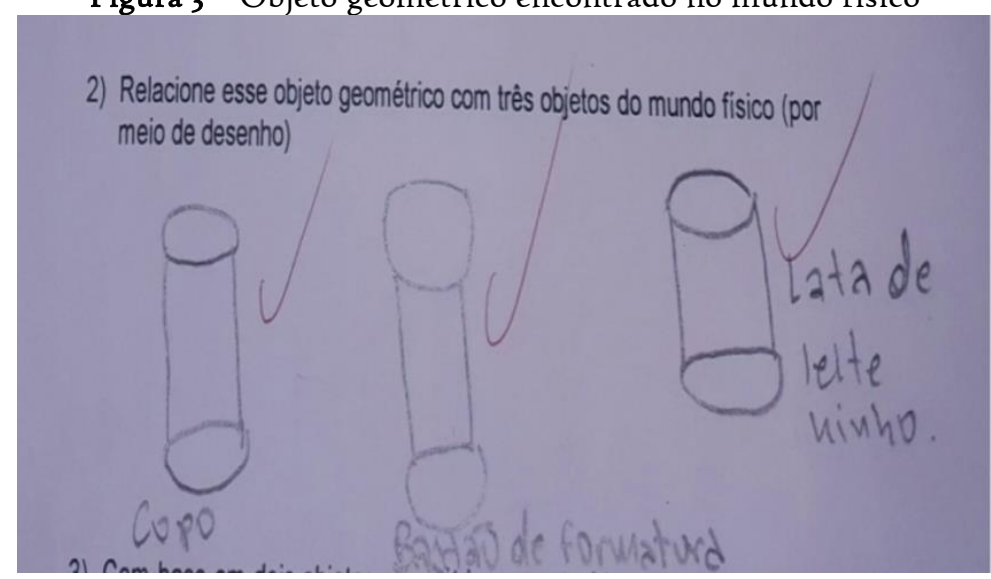

Refletir sobre essa atividade e sua aplicabilidade no âmbito escolar, no contexto da Etnomatemática, possibilita entender como a construção de conhecimentos matemáticos pode ser proposta a partir da utilização das experiências e vivências dos alunos, cuja intencionalidade permite produzir significados sobre a realidade em que estes sujeitos estão inseridos, e, por outro viés, possibilita reafirmar a apropriação de conceitos matemáticos, relacionados a Espaço e Forma, presente em Geometria e em acordo com os documentos balizadores do ensino desses saberes nos Anos Iniciais do Ensino Fundamental. Com isso, pode-se afirmar que a Etnomatemática se expressa como ferramenta essencial no aprendizado dos alunos dessa etapa de ensino, pois, conforme D’Ambrósio (2005, p. 114).

[...] Etnomatemática não é apenas o estudo de 'matemática das diversas etnias' [...] há várias maneiras, técnicas, habilidades (ticas) de explicar, de entender, de lidar e de conviver com (matema) distintos contextos naturais e socioeconômicos (etnos). 
Todavia, entendemos que a Etnomatemática, que surgiu na década de 1970 como proposta educacional de cunho sociocultural, possibilita um enfoque sobre a construção e produção de conhecimentos matemáticos, dialogando com questões sociais, culturais, antropológicas, históricos e filosóficos, o que permite uma discussão teórica e metodológica que transita entre os saberes populares e científicos, possibilitando ao aluno compreender o mundo ao seu redor através da Matemática e das demais ciências (AMBRÓSIO, 2005).

\section{CONSIDERAÇÕES FINAIS}

A disciplina Abordagens Teóricas-metodológicas da Matemática Escolar, do Curso de Pedagogia da UFPA, possibilitou a reflexão acerca das várias tendências de ensino que comporta o fazer docente, sobretudo, a Etnomatemática.

Em um curso de formação de professores que tem como objetivo formar profissionais qualificados e críticos diante dos distintos contextos sociais, com ênfase nos Anos Iniciais do Ensino Fundamental, foi possível fazer os discentes de Pedagogia apropriarem-se de teorias e práticas que contribuem para o processo de ensino e aprendizagem dos educandos dessa etapa de ensino, sendo a Matemática umas das disciplinas que requer dinamismo e domínio de conhecimentos por parte do professor sobre os assuntos que compõem os saberes matemáticos para melhor ensiná-los aos alunos.

Concordamos com o professor Ubiratan D' Ambrósio (2005) quando, em seus estudos, afirma que a Matemática dominante que está presente nos currículos educacionais, por vezes, suprimiu a Matemática do dia a dia. Entretanto, para além dessa concepção de superioridade, a produção e construção do saber matemático precisa ser concebida em uma visão holística do conhecimento, em uma perspectiva multicultural, intercultural e transdisciplinar de ensino que permeia o processo educativo, sendo a Etnomatemática uma tendência importante que utiliza desses elementos na produção do conhecimento matemático, seja de teor científico ou cultural da Matemática Escolar.

Todavia, a Matemática no âmbito da escola, embora com o avanço de técnicas e métodos de ensino, ainda persiste na ideia de uma ciência isolada e permeada de abstrações, que não tem conexões e não dialoga com o mundo físico e suas práticas sociais, desconsiderando que o conhecimento matemático tem uma linguagem própria em cada comunidade social, que permite ao sujeito interpretar e resolver situações problemas do cotidiano, pois a Matemática não se restringe apenas a abstrações, decoração de fórmulas e regras. Esses elementos necessitam fazer sentido na realidade e na vida prática desses sujeitos, algo que a Etnomatemática busca valorizar, 
dando espaço para que as conexões e complexidades que estão imbuídas nas vivências e experiências dos indivíduos sejam consideradas.

Ter compreensão acerca das teorias e métodos próprios para o ensino da Matemática é primordial para o professor em formação, especialmente para quando este estiver em sua atuação como docente em sala de aula, pois, o mesmo precisa ter subsídios, além de conhecimentos e métodos adequados, para que o cenário da educação, em especial da questão que envolve o ensino da matemática na escola, se mostre transformador, e dê sentido a ação e a reflexão do fazer pedagógico.

\section{REFERÊNCIAS}

BRASIL. Secretaria de Educação Fundamental. Parâmetros Curriculares Nacionais: matemática. Brasília: MEC/SEF, 1997.

BRASIL. Secretaria de Educação Básica. Base Nacional Curricular Comum. Brasília: $\mathrm{MEC} / \mathrm{SEB}, 2017$.

D’AMBRÓSIO, Ubiratan. Etnomatemática: arte ou técnica de explicar e conhecer. São Paulo: Ática, I99o.

D’AMBRÓSIO, Ubiratan. Sociedade, cultura, matemática e o seu ensino. Educação e Pesquisa, v. 31, n. I, p. 99-120, 2005.

FELIX, Edneia; AZEVEDO, Antulio José de. Geometria: como trabalhar os conceitos geométricos nas séries iniciais do Ensino Fundamental. Revista de Ciência Aplicada da FAIP, Marília, p. I-50, 2015.

FIORENTINI, Dario. Alguns modos de ver e conceber o ensino de matemática no Brasil. Zetetiké, Campinas, ano 3, n. 4, novembro de 1995, p. or-37.

HAGE, Salomão Mufarrej. Interculturalidade, Fratenidade e Comunhão: Referências para a sustentabilidade na Amazônia. Anais do XVII Congresso Eucarístico Nacional: Simpósio sobre a Amazônia. Belém/PA, 2016.

RODRIGUES, Luciano Lima. A Matemática ensinada na escola e a sua relação com o cotidiano. Brasília: Universidade Católica de Brasília, 2004.

Rodrigues, José Maria Soares. O uso de materiais manipuláveis no entendimento de alunos de Pedagogia: Estudos de Espaço e Forma. Anais do VI Congresso Nacional de Educação: CONEDU. 2019.

SMOLE, Katia Stocco. Alfabetização Matemática: implicações para o ensino e aprendizagem da Matemática Escolar. São Paulo: Suplemento Pedagógico APASE, 2012. 